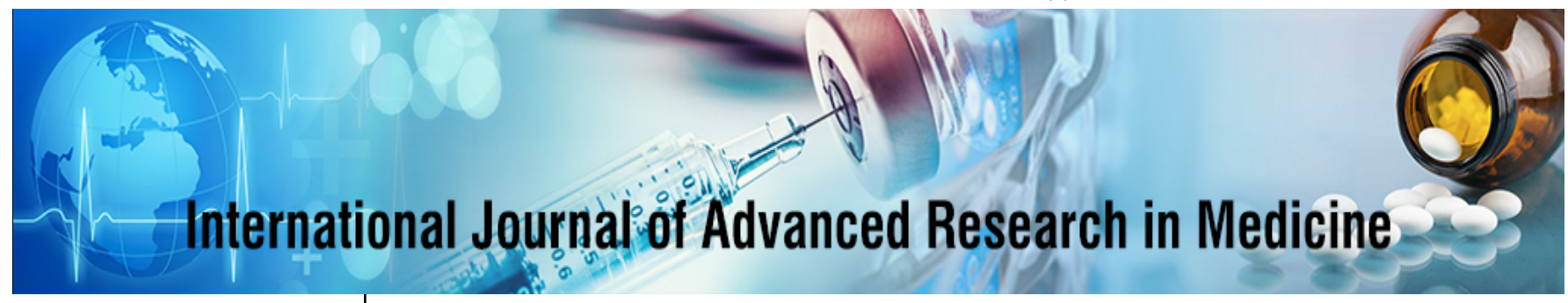

E-ISSN: 2706-9575

P-ISSN: 2706-9567

IJARM 2021; 3(2): 158-164

Received: 06-07-2021

Accepted: 28-07-2021

Dr. Mohammed Azam

Associate Professor, I/C HOD, Department of Paediatrics,

Govt Medical College

Nalgonda, Telangana, India

\section{Observational study on clinical profile and outcome of Rickettsial infections in children}

\author{
Dr. Mohammed Azam
}

DOI: https://doi.org/10.22271/27069567.2021.v3.i2c.237

\begin{abstract}
Background: Rickettsial diseases are one of the most re-emerging infections of the present time. They are generally incapacitating and difficult to diagnose. Untreated cases can have fatality rates as high as $30-35 \%$ but when diagnosed properly, they are often easily treatable. Rickettsial infections are one of the important causes of pyrexia of unknown origin (PUO) and this needs to be differentiated from other febrile illnesses. Rickettsial infections are grossly under- diagnosed in India.

Objectives of The Study: To study the clinical profile and outcome of rickettsial infections in children aged less than 12 years

Methods: It is a time bound prospective hospital based observational study conducted from 2019 to 2020. All children aged less than 12 years admitted in paediatric ward with fever without an identifiable source of infection and one or more of the following clinical features: rash, oedema, hepatosplenomegaly, Lymphadenopathy, an Eschar and a tick bite or tick exposure were suspected to have rickettsial infection. The purpose of the study was explained to the parents or guardians of the child and an informed consent was taken from them enrolling the child in study group. All suspected cases were subjected to rickettsial IgM/IgG ELISA test and tests to exclude other diseases.All rickettsial IgM/IgG positive cases were followed up through hospital stay and outcomes were noted.

Results: The most common age group of presentation was between 1 and 5 yrs. The common symptoms in these children included fever (100\%), rash (83.3\%), edema of limbs (26.6\%), puffiness of face (30\%), generalised edema (23.3\%), cough (23.3\%), pain abdomen (16.6\%), vomiting (13.3\%), convulsions (10\%), headache (3.33\%) and arthralgia (3.33\%). Signs like Hepatomegaly, facial puffiness, pedal edema, splenomegaly, ecchymosis present in $53.3 \%, 46.6 \%, 43.3 \%, 10 \%$ and $6.6 \%$ of the cases respectively, mimicking common illnesses. Thus warrants high index of suspicion. SCRUB Typhus and Indian Tick Typus Elisa Positive (mixed infection) noted in 14\% of cases. Complications like meningoencephalitis (3.33\%), shock (3.33\%), DIC (3.33\%) was observed in the study. There was good clinical response on initiation of doxycycline within $48 \mathrm{hrs}$ of initiation of treatment. Case fatality rate of rickettsial disease in this study was $6.66 \%(n=2)$.

Conclusion: Indian tick typhus is the most common rickettsial fever noted in this part of Telangana. Rickettsial diseases are difficult to diagnose, unless suspected but treatment is easy, affordable and often successful with dramatic response to antimicrobials.
\end{abstract}

Keywords: Rickettsial infection, ELISA, Childrens, Scrub Typhus, Hepatomegaly, Facial Puffiness.

\section{Introduction}

Zinc is an essential trace element important for almost all biological systems. Zinc nutritive is likely to be suboptimal in many children of developing countries and may contribute to their impaired growth, increased susceptibility to infections and possibly to the high mortality. A large proportion of childhood deaths in developing countries are caused by diarrhea and pneumonia. Impaired zinc nutritive seems to play a role in the increased incidence and severity of these infections ${ }^{[1,2]}$.

Acute lower respiratory infections (ALRI) predominantly pneumonia cause approximately4 million deaths every year, accounting for one-third of all childhood deaths in developing countries ${ }^{[1]}$. Various factors have been associated with acute respiratory infections (ARI) in general and pneumonia in particular. These include, among others, nutritional status [2, 3, 4], family characteristics ${ }^{[5]}$ and environmental exposures ${ }^{[7,8]}$. Most of environmental risk factors require multispectral coordination for modification. In contrast, some of the childhood risk factors can be modified by simple interventions like vitamin A or zinc supplementation ${ }^{[13]}$. Recent works have provided conflicting ${ }^{[9,10,11]}$ evidence on the role of Rickettsial diseases are one of the most re-emerging infections of the present time, which are
Dr. Mohammed Azam Associate Professor, I/C HOD, Department of Paediatrics, Govt Medical College Nalgonda, Telangana, India 
often failed to diagnose ${ }^{[1]}$. Untreated cases can have fatality rates as high as $30-35 \%$ but when diagnosed properly, they are often easily treatable ${ }^{[2]}$. It is one of the most important causes of pyrexia of unknown origin (PUO) and this needs to be differentiated from other febrile illnesses like Enteric fever, Malaria, Dengue, Infectious mononucleosis, Kawasaki disease, Collagen vascular diseases ${ }^{[1]}$. Rickettsial infection is a zoonotic acute febrile illness caused by small, non-flagellated, obligate intracellular, pleomorphic gramnegative coccobacilli from the genera Rickettsia, Orientia, Ehrlichia, Neorickettsia and Anaplasma and they are transmitted by arthropod vectors. They are primary parasites of arthropods like lice, fleas, ticks and mites, in which they are found in the alimentary canal ${ }^{[3]}$.

Rickettsial infections are grossly under-diagnosed in India. As no single laboratory test is specific for early diagnosis, treatment needs to be started empirically on clinical and epidemiological suspicion. In view of low index of suspicion, nonspecific signs and symptoms, and absence of widely available sensitive and specific diagnostic test, these infections are extremely difficult to diagnose but treatment is easy, affordable and often successful with dramatic response to antimicrobials ${ }^{[1]}$.

The National Centre for Disease Control (NCDC, formerly National Institute of Communicable Disease) has played important role in providing serological evidence of rickettsial diseases in India in various States like Jammu\& Kashmir, Himachal Pradesh, Uttarakhand, Haryana, Rajasthan, Assam, West Bengal, Maharashtra, Tamil Nadu, Kerala, Karnataka, Sikkim, and Manipur in the last decade ${ }^{[4]}$. Batra has reported high magnitude of scrub typhus, spotted fever Indian tick typhus ${ }^{[2]}$.

Weil-Felix antibody testing should not be performed, because it lacks both sensitivity and specificity. Definitive diagnosis is most often accomplished by serology, which is retrospective, because a rise in titre is not seen until after the first week of illness. Orientia tsutsugamushi serologic tests such as indirect fluorescent antibody assay are approximately $90 \%$ sensitive with 11 or more days of fever. Infections from Indian tick typhus can be confirmed by immunohistologic or immunofluorescent or demonstration of seroconversion by 4 fold rise in serum antibody titres ${ }^{[4]}$. IgM and IgG ELISA: ELISA techniques, particularly immunoglobulin $\mathrm{M}$ (IgM) capture assays are probably the most sensitive tests available for rickettsial diagnosis and the presence of IgM antibodies, indicates recent infection with rickettsia. Immunufluoroscence assay (IFA) is a gold standard for diagnosis of rickettsial infection ${ }^{[5]}$.

The majority of studies regarding rickettsial infections in India and other parts of the world are based on adult populations and on scrub typhus. There is a paucity of studies regarding the incidence and clinical profile of rickettsial disease in children from the Indian subcontinent and majority of published studies are retrospective or sporadic case reports ${ }^{[6]}$. Hence this study aims to study the clinical features and outcomes of paediatric rickettsial disease.

\section{Objectives}

To study the clinical profile and outcome of rickettsial infections in children aged less than 12 years.

\section{Methodology \\ Source of data}

All children less than 12 years of age with clinical features of Rickettsial infection and serology positive for Rickettsial
IgM/IgG ELISA admitted to Govt Medical College \& General Hospital Nalgonda, Telangana, India.

\section{Method of collection of data}

Study design: Prospective time bound observational study.

\section{Inclusion criteria}

- All children up to 12 years of age will be considered.

- All children aged less than 12 years admitted in pediatric ward with fever without an identifiable source of infection and one or more of the following clinical features: rash, edema, hepatosplenomegaly, lymphadenopathy, an eschar and a tick bite or tick exposure were suspected to have rickettsial infection along with positive serology test for Rickettsial IgM/IgG ELISA

\section{Exclusion criteria}

- Cases with other established causes of infection

\section{Method of Study}

It is a time bound prospective hospital based observational study conducted from 2019 to 2020. All children aged less than 12 years admitted in pediatric ward with fever without an identifiable source of infection and one or more of the following clinical features: rash, edema, hepatosplenomegaly, lymphadenopathy, an eschar and a tick bite or tick exposure were suspected to have rickettsial infection. The purpose of the study was explained to the parents or guardians of the child and an informed consent was taken from them enrolling the child in study group. All suspected cases were subjected to rickettsial IgM/IgG ELISA test and tests to exclude malaria- thick and thin peripheral smear, WIDAL for enteric fever and NS 1 and IgM Mac ELISA for dengue fever. Patients were treated according to the hospital protocol. All rickettsial IgM/IgG positive cases were followed up through hospital stay and outcomes were noted. The ELISA kit used in this study was Scrub typhus IgM/IgG from INBIOS international from USA and Rickettsia Conorii ELISA IgM/IgG VIRCELL from Spain.

Chest X-rays, tests for renal and liver function, serum electrolyte, ultrasonography (USG), neuroimaging, cerebrospinal fluid analysis (CSF) were done as and when required.

\section{Data Collection}

A predesigned proforma was used to collect data regarding age, gender, residential area, exposure to animals. Clinical data, including the duration of the fever, associated symptoms, vital signs, general and systemic examination. findings, complications, investigations, treatment and defervescence time were recorded.

\section{Scrub Typhus IGM Elisa System}

The Scrub Typhus IgM ELISA test for exposure to Orientia tsutsugamushi (OT) is an ELISA assay system for the detection of IgM antibodies in human serum to OT derived recombinant antigen. This test is to aid in the diagnosis of human exposure to OT species.

\section{Kit performance and quality}

It has got a sensitivity of $91 \%$ and Specificity of $100 \%$.

\section{Data analysis}

Results are determined by strength of average OD values for a given sample. Cut-off value in our study was 0.2652 . 


\section{Rickettsia Conorii Elisa IgG/IgM \\ Principle of the test}

The ELISA method is based upon the reaction of antibodies in the sample tested with the antigen adsorbed on the polystyrene surface. Unbound immunoglobulins are washed off. An enzyme-labeled anti-human globulin binds the antigen-antibody complex in a second step. After a new washing step, bound conjugate is developed with the aid of a substrate solution (TMB) to render a blue colored soluble product which turns into yellow after adding the acid stopping solution.

\section{Interpretation of results}

Calculated the mean O.D. for cut off serum.

Antibody index = (sample O.D./ cut off serum mean O.D.) $\mathrm{x}$ 10.

Table 1: Kit Performance and Quality

\begin{tabular}{|c|c|c|}
\hline & Sensitivity & Specificity \\
\hline Ig M & $94 \%$ & $95 \%$ \\
\hline Ig G & $85 \%$ & $100 \%$ \\
\hline
\end{tabular}

Table 2: Interpretation of the Result

\begin{tabular}{|c|c|}
\hline Index & Interpretation of the Result \\
\hline$<9$ & Negative \\
\hline $9-11$ & Equivocal \\
\hline$>11$ & Positive \\
\hline
\end{tabular}

- Meningoencephalitis was diagnosed when the following conditions were observed-

i). Encephalopathy features like altered sensorium, seizures, meningismus

ii). CSF analysis suggestive of aseptic meningitis

iii). Neuroimaging findings suggestive of meningoencephalitis ${ }^{4}$

- Hypotension was defined as a Systolic blood pressure below the 5th percentile for the corresponding age, sex and height.

- Hepatitis was diagnosed when liver transaminases were found to be elevated (twice the normal range) ${ }^{7}$

- Thrombocytopenia was defined as a platelet count of less than 150,000/cumm ${ }^{7}$

- Vasculitis was diagnosed when gangrene of the extremities or purpura fulminans present.

- Bronchopneumonia was diagnosed with streaky opacities or parenchymal lung lesions on a chest radiograph and presence of cough or dyspnea.

- Disseminated intravascular coagulation was diagnosed; spontaneous bleeding, thrombocytopenia, prolonged PT/aPTT.

\section{Statistical Analysis}

Data was analysed in Microsoft Office Excel 2007 version. Descriptive statistics are presented in terms of counts, percentages, mean and standard deviation values.

\section{Results}

Table 3: Elisa for Rickettsial IGM/igg ( $n=30)$

\begin{tabular}{|c|c|c|}
\hline Elisa & Positive & Percentage \\
\hline Scrub typhus & 5 & $16.6 \%$ \\
\hline Indian tick typhus & 20 & $66.6 \%$ \\
\hline Scrub typhus and indian tick ty-Pus (mixed) & 5 & $16.6 \%$ \\
\hline
\end{tabular}

Out of 30 cases positive for rickettsial IgM/IgG 5(16.6\%) were positive for scrub typhus, 20(66.66\%) and 5(16.6\%) were positive for both scrub and Indian tick ty- phus.

Table 4: Gender Distribution $(n=30)$

\begin{tabular}{|c|c|c|}
\hline Gender & No. of Patients & Percentage (\%) \\
\hline Male & 12 & 40 \\
\hline Female & 18 & 60 \\
\hline Total & 30 & 100 \\
\hline
\end{tabular}

Out of 30 children diagnosed with rickettsial disease, 40\% (12) were boys and $60 \%$ (18) were girls.

Table 5: Age Group Wise Distribution of Cases $(n=30)$

\begin{tabular}{|c|c|c|}
\hline Age in years & No. of Patients & Percentage (\%) \\
\hline$<1$ year & 2 & 6.66 \\
\hline $1-5$ years & 15 & 50 \\
\hline $6-10$ years & 8 & 26.6 \\
\hline $11-12$ years & 5 & 16.6 \\
\hline Total & 30 & 100.0 \\
\hline
\end{tabular}

Most common age group of occurrence of rickettsial infection was between 1 to 5 years (50\%) followed by the age group of 6 to 10 years with 8 cases (26.6\%).

Table 6: Monthwise Distribution of Cases $(n=30)$

\begin{tabular}{|c|c|c|}
\hline Month & No. of Patients & Percentage (\%) \\
\hline January & 1 & 3.33 \\
\hline February & 1 & 3.33 \\
\hline March & 1 & 3.33 \\
\hline May & 2 & 6.66 \\
\hline June & 2 & 6.66 \\
\hline July & 3 & 10 \\
\hline August & 6 & 20 \\
\hline September & 4 & 13.3 \\
\hline October & 3 & 10 \\
\hline November & 4 & 13.3 \\
\hline December & 3 & 10 \\
\hline
\end{tabular}

More cases presented during second half of the year. Maximum number of cases were in the month of August $(n=6,20 \%)$, followed by September $(n=4,13.3 \%)$. 
Table 7: Clinical Symptoms ( $\mathrm{n}=30)$

\begin{tabular}{|c|c|c|c|}
\hline & Symptoms & No. of patients $(n=34)$ & $\%$ \\
\hline & Fever & 30 & 100 \\
\hline & Duration $<5$ days & 2 & 6.66 \\
\hline Duration of fever & 5-7 days & 18 & 60 \\
\hline & 8-10 days & 10 & 33.3 \\
\hline & Generalized rash & 25 & 83.3 \\
\hline Rasn & Rash with involvement of palms and soles & $15 / 25$ & 60 \\
\hline & Edema of legs & 8 & 26.66 \\
\hline & Facial puffiness & 9 & 30 \\
\hline & Generalized edema & 7 & 23.33 \\
\hline & Cough & 7 & 23.33 \\
\hline & Pain abdomen & 5 & 16.66 \\
\hline & Vomiting & 4 & 13.33 \\
\hline & Convulsion/ Seizures & 3 & 10 \\
\hline Cont & with pets or exposure to an-imals & 10 & 33.3 \\
\hline & Headache & 1 & 3.33 \\
\hline & Arthralgia & 1 & 3.33 \\
\hline
\end{tabular}

Fever was present in all cases $(n=30,100 \%)$. Duration of fever ranged from 2 days to 10 days, of which 2 cases (6.66\%) presented within 4 days of illness. $60 \% \quad(n=18)$ presented in the second half of first week. 33.3\% $(n=10)$ presented in the first half of second week.

Rash was seen in $83.3 \%(n=25)$ of cases. Out of those 27 cases 17 had involve- ment of palms and soles (60\%). The other common presentation was symptoms were periorbital puffiness in $30 \%(n=9)$, edema of legs in $26.6 \%(n=8)$, gene- ralized edema in $20.6 \%(n=7)$.

Contact with pets or animal exposure: was seen in 12(35.2\%) cases.

Table 8: Clinical Signs ( $n=30)$

\begin{tabular}{|c|c|c|c|c|c|}
\hline & & \multicolumn{2}{|c|}{ No. of patients $(n=30)$} & \multicolumn{2}{|c|}{$\%$} \\
\hline \multirow{3}{*}{ Rashes } & Macular & \multirow{3}{*}{28} & \begin{tabular}{|l|}
5 \\
\end{tabular} & \multirow{3}{*}{93.3} & \multirow{2}{*}{$\frac{17.8}{46.4}$} \\
\hline & Maculo-papular & & 13 & & \\
\hline & Petechiae & & 10 & & 35.7 \\
\hline & Hepatomegaly & & 16 & & 3.3 \\
\hline & Facial Puffiness & & 14 & & .66 \\
\hline & Pedal Edema & & 13 & & .33 \\
\hline & onjunctival congestion & & 9 & & 0 \\
\hline & Pallor & & 7 & & .33 \\
\hline & Ascites & & 5 & & 5.6 \\
\hline & Generalized Edema & & 4 & & .33 \\
\hline & Hepatosplenomegaly & & 3 & & 0 \\
\hline & Ecchymosis & & 2 & & 66 \\
\hline
\end{tabular}

Out of 34 cases, rash was present in 28 cases (82.3\%). $53.3 \%(n=16)$ cases presented with hepatomegaly. Facial Puffiness was present in $46.6 \%(n=14) .43 .3 \%(n=13)$ cases were present with pedal Edema and $29.4 \%(n=10)$ cases were present with petechiae. Conjunctival congestion was present in $30 \%(n=9)$ of children and pallor in $23.3 \%(n=7)$. Signs of ascites were present in 5 children (16.6\%). Generalized Edema was present in $23.3 \%(n=7)$ of children. Hepatosple- nomegaly was seen in $10 \%(n=3)$. Signs of Ecchymosis were present in 2 child- ren (6.66\%).

\section{Laboratory Parameters}

Table 9: Blood Parametres

\begin{tabular}{|c|c|c|c|}
\hline & Parameters & No. of patients (n) & $\%$ \\
\hline \multicolumn{4}{|c|}{ Hemoglobin(g/dl) } \\
\hline & $<9$ & 7/30 & 23.3 \\
\hline - & $>9$ & $25 / 30$ & 83.3 \\
\hline \multicolumn{4}{|c|}{ TLC (cumm) } \\
\hline & Normal & $25 / 30$ & 83.3 \\
\hline & Leucopenia & $3 / 30$ & 10 \\
\hline$\cdot$ & Leukocytosis & $4 / 30$ & 13.3 \\
\hline \multicolumn{4}{|c|}{ Platelet counts } \\
\hline & $<1.5$ lakh/cumm & $10 / 30$ & 33.3 \\
\hline - & $>1.5$ lakh/cumm & $20 / 30$ & 66.6 \\
\hline \multicolumn{4}{|c|}{ S. Sodium (mEq/L) } \\
\hline & Normal & $8 / 30$ & 26.6 \\
\hline
\end{tabular}




\begin{tabular}{|c|c|c|c|}
\hline$\bullet$ & Hyponatremia & $20 / 30$ & 66.6 \\
\hline \multicolumn{4}{|c|}{ S. AST(U/L) } \\
\hline & Normal & $10 / 15$ & 66.6 \\
\hline & Elevated & $4 / 15$ & 26.6 \\
\hline \multicolumn{4}{|c|}{ S. ALT(U/L) } \\
\hline & Normal & $12 / 15$ & 80 \\
\hline & Elevated & $4 / 15$ & 26.6 \\
\hline \multicolumn{4}{|c|}{ Total Bilirubin (mg/dl) } \\
\hline & Normal & $15 / 15$ & 100 \\
\hline & Elevated & $1 / 15$ & 6.66 \\
\hline \multicolumn{4}{|c|}{ Total protein $(\mathrm{gm} / \mathrm{dl})$} \\
\hline & Normal & $11 / 15$ & 73.33 \\
\hline & Decreased & $5 / 15$ & 33.3 \\
\hline \multicolumn{4}{|c|}{ Albumin (gm/dl) } \\
\hline & Normal & $14 / 15$ & 93.33 \\
\hline & Decreased & $2 / 15$ & 13.33 \\
\hline \multicolumn{4}{|c|}{ S. Creatinine(mg/dl) } \\
\hline & Normal & $30 / 30$ & 100 \\
\hline
\end{tabular}

Considering hematological parameters, 23.3\% ( $\mathrm{n}=7$ ) had hemoglobin $<9 g \%$, 10\% $(n=3)$ had leucopenia and 13.3\% $(\mathrm{n}=4)$ had leukocytosis. Thrombo- cytopenia $(<1.5$ lakh/cumm) was present in 10 cases (33.3\%).

Renal function test, liver function tests, were done as and when required. Out of 30 cases, 8 cases had normal serum sodium levels. Hyponatremia (S. Sodium $<135 \mathrm{mEq} / \mathrm{L}$ ) was present in 20 cases $(66.6 \%) .4$ out of 15 cases had ele- vated serum transaminase level (AST and ALT elevated twice the normal value).

Elevated bilirubin $(>2 \mathrm{mg} / \mathrm{dl})$ was present in 1 out of 15 cases. Hypoalbuminemia was seen in 2 out of 15 cases. 25 out of 30 cases had renal function test done, all had normal serum creatinine levels.

\section{Imaging}

Two cases had neuroimaging (CT Brain) done, both reported normal. USG was done on 18 children. One children enlarged hypoechoic liver with splenome- galy and one children had hepatosplenomegaly. Sixteen children USG Abdomen scanning was normal.

CSF analysis was done in only one patient, which showed features of aseptic meningitis (Cell count 20 cells, Cell type $70 \%$ lymphocytes $30 \%$ neutro- phils, glucose-59, protein-22 and chloride-98. ZN stain-Neg, Gram stain-Neg, C/S -no growth)

\section{Complications}

Out of 30 cases, 3 cases had complications.

- One patient had meningoencephalitis with Shock.

- Rickettsial vasculitis with DIC with Shock was seen in one case and

- Meningoencephalitis was seen in one case.

Table 10: Duration of Hospitalization $(n=30)$

\begin{tabular}{|c|c|c|}
\hline Duration & No. of Patients & $\mathbf{\%}$ \\
\hline$\leq 5$ days & 15 & 50 \\
\hline $6-10$ days & 14 & 46.6 \\
\hline$\geq 11$ days & 1 & 3.33 \\
\hline Total & 30 & 100.0 \\
\hline
\end{tabular}

Out of 30 cases, 15 cases (50\%) had duration of hospitalization for less than or equal to 5 days, 14 cases
(46.6\%) were hospitalized for 6-10 days and 1 cases (3.33\%) for 21 days as child had meningoencephalitis.

Table 11: Outcome $(\mathrm{n}=30)$

\begin{tabular}{|c|c|c|}
\hline Outcome & No. of patients & \% \\
\hline Died & 1 & 3.33 \\
\hline Improved & 29 & 96.3 \\
\hline Total & 30 & 100.0 \\
\hline
\end{tabular}

Out of 30 cases 1 case died accounting for a case fatality rate of $3.33 \%$. Cause of death was

1. Meningoencephalitis with Shock.

2. Rickettsial vasculitis with DIC with Shock

\section{Discussion}

Rickettsial diseases are reported from various parts of India [1], However, the reported cases underestimate the burden of rickettsial diseases in India due to the lack of both community based studies and availability of specific laboratory tests. Present study describes the clinical profile of rickettsial infection in children.

\section{Demographic data}

The mean age of occurrence of rickettsial infection in present study was 5.4 years with maximum incidence in the age group of 1 to 5 yrs (50\%). Similar observations of age predilection for less than 5 years was made by Patil D et al., [8].

In this study females (60\%) outnumbered males, with male to female ratio of $0.9: 1$ (16:18). This differs from the studies by other authors like Kumar M et al., ${ }^{[12]}$. who reported male preponderance.

The majority of the cases were observed during second half of the year, from July to December around $79.5 \%$. Similar observations have been recorded by Krishna MR et al. ${ }^{9}$ (June to November).

In this study all the cases were from rural areas and most of them belonged to lower socioeconomic status. This might be attributable to more chances of over-crowding, poor hygiene, tick infestation and louse infestation in these patients. 


\section{Clinical profile \\ Clinical symptoms}

In the present study all patients presented with fever (100\%), similar finding was noted in other studies ${ }^{[10]}$. approximately $60 \%(n=18)$ of the patients presented with 57 days of acute febrile illness with no foci of infection. Rash was seen in $83.3 \%$ of which $60 \% \quad(n=15 / 25)$ had involvement of palms and soles. Facial puffiness was seen in $30 \%$, swelling of limbs seen in $26.6 \%$ and generalized edema in $23.3 \%$.

A history of tick/mite bite was not available in any of the cases. Similar findings have been reported by Sirisanthana V et al. ${ }^{[11]}$ None of the cases in the present study had eschar unlike other studies ${ }^{[12]}$. Mahajan SK et al. ${ }^{[6]}$ did not find eschar in any of their cases. Though presence of eschar confirms and is pathognomonic of scrub typhus group of rickettsial diseases, but its absence does not exclude the possibility of scrub typhus.

\section{Laboratory parameters}

In our study $10 \%(n=3)$ had leucopenia. Similar findings was noted in other studies like Takhar et al. ${ }^{[13]}$ (15\%).

Out of 30 cases, 8 cases had normal serum sodium levels. Hyponatremia (S.Sodium $<135 \mathrm{mEq} / \mathrm{L}$ ) was present in 20 cases (66.6\%). Similar findings were noted by Ratageri VH et al. ${ }^{[10]}(71.4 \%)$

\section{Complications}

The most common complication observed in this study was meningoencephalitis in 3.3\% (1) of cases. Similar findings were noted in studies by Rathi NB et al. ${ }^{[14]}$ and Palanivel S et al. ${ }^{[15]}$ reporting meningoencephalitis in $5 \%$ and $6 \%$ of patients respectively.

There were no renal or cardiac complication seen in this study, unlike study done by Kumar $\mathrm{M}$ et al. ${ }^{[12]}$ where in myocarditis with cardiogenic shock in 34\%, acute kidney injury in $20 \%$ of cases noted which was attributed to late referrals.

\section{Treatment}

All the 30 cases in our study received treatment with Doxycycline, of which 28 cases(93.3\%) was improved. 80\% responded to the treatment within $48 \mathrm{hrs}$ and $15 \%$ of cases responded after 48 hrs. Kumar $\mathrm{M}$ et al. ${ }^{[12]}$ has demonstrated that good response to Doxycycline (97\%).

\section{Outcome}

Rickettsial disease is known to produce serious complications and has a mortality rate of $30 \%{ }^{[2,4]}$. Deaths were attributable to late presentation, delayed diagnosis and drug resistance.

\section{Conclusion}

Rickettsial diseases are one of the important causes of pyrexia of unknown origin, which are grossly under diagnosed in India.

It is most commonly seen in younger age group. Children presenting with fever, rash, edema of legs or facial puffiness should be suspected of rickettsial fever. Hepatosplenomegaly, rash and third space fluid loss are the most common clinical signs noted. Rickettsial diseases are difficult to diagnose, unless suspected but treatment is easy, affordable and often successful with dramatic response to antimicrobials.

\section{Acknowledgment}

The author is thankful to Department of Pediatrics Govt Medical College\& General Hospital Nalgonda for providing all the facilities to carry out this work.

\section{Conflict of Interest}

None

\section{Financial Support}

Nil

\section{References}

1. Rathi N, Rathi A. Rickettsial infections Indian perspective. Ind Ped 2010;47:157-164

2. Batra HV. Spotted fevers and typhus fever in Tamil Nadu- commentary. Indian J Med Res 2007;126:101103.

3. Jayaram Paniker CK. Ananthanarayan and Paniker's Textbook of Microbiology. $7^{\text {th }}$ ed. University Press Pvt Ltd 2008, 412-421.

4. Reller ME, Dumler JS. Scrub Typhus (Orientia tsutsugamushi). In: Kliegman RM, Stanton BF, GemeIII JWS, Schor NF, Behrman RE, editors. Kliegman: Nelson Textbook of Pediatrics. First south asia ed: Saunders, An Imprint of Elsevie 2011.

5. World Health Organization. WHO Recommended Surveillance Standards, WHO/CDS/CSR/ISR/99.2, Geneva, Switzerland.

6. Mahajan SK, Rolain JM, Sankhyan N, Kaushal RK, Raoult D. Pediatric Scrub typhus in Indian Himalayas. Indian J Pediatr 2008;75:947-949.

7. Issac R, Varghese GM, Mathai E, Manjula J, Joseph I. Scrub typhus: Prevalence and diagnostic issues in rural Southern India. Clin Infect Dis 2004;39:1395-1396.

8. Patil Deepa, Bidari LH, Tikare N, Revankar VA. Profile of rickettsial fever in children. In: 2006 e Conference Abstracts. Pediatric Oncall [serial online]. Karnataka Pedicon; 2006 [cited 15 November 2006;(Supplement 11)];3. Available from http:/www.pediatriconcall.com/fordoctor/conference_a bstracts/Karnataka_Pedicon/.

9. Krishna MR, Vasuki B, Nagaraju K. Scrub typhus: audit of an outbreak. The Indian Journal of Pediatrics 2015;82(6):537-40.

10. Ratageri VH, Madhu PK, et al. Clinico-laboratory profile and outcome of rickettsia in children: Hubli (Karnataka) experience. Paediatric infectious disease 2014;(6):3-6.

11. Sirisanthana V, Puthanakit T, Sirisanthana T. Epidemiologic, clinical and laboratory features of scrub typhus in thirty Thai children. The Pediatric infectious disease journal 2003;22(4):341-5.

12. Kumar M, Krishnamurthy S, Delhikumar CG, Narayanan P, Biswal N, Srinivasan S. Scrub typhus in children at a tertiary hospital in southern India: Clinical profile and complications. J Infect Public Health 2012;5(1):82-8.

13. Takhar RP, Bunkar ML, Arya S, Mirdha N, Mohd A. Scrub typhus: A prospec tive, observational study during an outbreak in Rajasthan, India. The National Medical Journal of India 2017;30(2):

14. Rathi NB, Rathi AN, Goodman MH, Aghai ZH. Rickettsial diseases in central India: proposed clinical 
scoring system for early detection of spotted fever. In dian pediatrics 2011;48(11):867-72.

15. Palanivel S, Nedunchelian K, Poovazhagi V, Raghunadan R, Ramachandran P. Clinical profile of scrub typhus in children. The Indian Journal of Pediatrics 2012;79(11):1459-62. 